\title{
Culture- and metagenomics-enabled analyses of the Methanosphaera genus reveals their monophyletic origin and differentiation according to genome size
}

\author{
Emily C. Hoedt ${ }^{1}$ - Donovan H. Parks ${ }^{2,3}$ - James G. Volmer ${ }^{1,3}$ - Carly P. Rosewarne ${ }^{4}$ - Stuart E. Denman ${ }^{5}$. \\ Christopher S. McSweeney ${ }^{5}$ - Jane G. Muir ${ }^{6}$ Peter R. Gibson ${ }^{6}$ - Páraic Ó Cuív ${ }^{1}$ - Philip Hugenholtz $\mathbb{D}^{1,2,3}$. \\ Gene W. Tyson ${ }^{2,3} \cdot$ Mark Morrison $^{1,2}$
}

Received: 27 November 2017 / Revised: 27 April 2018 / Accepted: 3 June 2018 / Published online: 1 August 2018

(c) International Society for Microbial Ecology 2018

\begin{abstract}
The genus Methanosphaera is a well-recognized but poorly characterized member of the mammalian gut microbiome, and distinctive from Methanobrevibacter smithii for its ability to induce a pro-inflammatory response in humans. Here we have used a combination of culture- and metagenomics-based approaches to expand the representation and information for the genus, which has supported the examination of their phylogeny and physiological capacity. Novel isolates of the genus Methanosphaera were recovered from bovine rumen digesta and human stool, with the bovine isolate remarkable for its large genome size relative to other Methanosphaera isolates from monogastric hosts. To substantiate this observation, we then recovered seven high-quality Methanosphaera-affiliated population genomes from ruminant and human gut metagenomic datasets. Our analyses confirm a monophyletic origin of Methanosphaera spp. and that the colonization of monogastric and ruminant hosts favors representatives of the genus with different genome sizes, reflecting differences in the genome content needed to persist in these different habitats.
\end{abstract}

Electronic supplementary material The online version of this article (https://doi.org/10.1038/s41396-018-0225-7) contains supplementary material, which is available to authorized users.

Mark Morrison

m.morrison1@uq.edu.au

1 The University of Queensland Diamantina Institute, Translational Research Institute, Brisbane, Queensland, Australia

2 Australian Centre for Ecogenomics, The University of Queensland, St Lucia, Australia

3 School of Chemistry and Molecular Biosciences, The University of Queensland, St Lucia, Queensland, Australia

4 Commonwealth Scientific and Industrial Research Organisation, Kintore Avenue, Adelaide, Australia

5 Commonwealth Scientific and Industrial Research Organisation, Queensland Bioscience Precinct, St Lucia, Australia

6 Department of Gastroenterology, Central Clinical School, The Alfred Centre, Monash University, Melbourne, Victoria, Australia

\section{Introduction}

Methanogenic archaea colonize a variety of anoxic habitats including the gastrointestinal tracts of warm-blooded animals, where they are widely acknowledged to contribute to host digestive function via their key role in coordinating interspecies hydrogen transfer and promoting more efficient growth of heterotrophic fermentative bacteria [1-3]. Generally, the principal members of these gut methanogenic communities are the Methanobacteriales (i.e., Methanobrevibacter and Methanosphaera spp.). Methanobrevibacter spp. are often the numerically dominant members of these taxocenes, favoring hydrogen-dependent reduction of carbon dioxide and/or formate to methane. In contrast, much less is known about the methylotrophic archaea, such as Methanomassiliicoccales and Methanosphaera spp. Draft genomes have been produced for several members of the Methanomassiliicoccales, and their role in livestock methane emissions [4, 5] and methylamine metabolism established [6-10].

Despite its discovery in the mid-1980s, Methanosphaera spp. have received relatively scant attention, with virtually all our knowledge derived from a single isolate recovered from human stool (Methanosphaera stadtmanae DSMZ3091 ${ }^{\mathrm{T}}$, 
[11]). Thauer et al. [12] proposed that energy conservation and hydrogen-dependent methanol reduction to methane by Methanosphaera stadtmanae is achieved without cytochromes, and involves electron bifurcation between the MvhADG-HdrABC and Ehb complexes that creates a coupling between ferredoxin and CoM-S-S-CoB reduction; this mode of electron bifurcation has recently been established biochemically by Yan et al. [13]. Hydrogen-fueled methanogenesis is also conserved in Methanosphaera cunculii $\operatorname{DSMZ4103}^{\mathrm{T}}[14,15]$. The physiological consequences of this mechanism of methanogenesis and energy conservation is consistent with recent findings that populations of Methanosphaera spp. are remarkably high in "low hydrogen/methane" producing ruminants [16]. However, their metabolic versatility may be more expansive than previously appreciated as we recently reported the isolation and characterization of a new member of the genus Methanosphaera (strain WGK6) from the foregut microbiome of the Western Grey Kangaroo (Macropus fulgidus) that is capable of using either hydrogen or ethanol as a source of reducing power for methanogenesis and growth [17].

In humans, Methanosphaera spp. also appear to be implicated with the onset of digestive diseases, and furthermore, stimulate various arms of the immune system. Blais Lecours et al. [18] reported greater absolute counts and prevalence of Methanosphaera spp. to occur in patients with inflammatory bowel disease (IBD), compared with healthy non-IBD subjects. The IBD patients were also found to possess high titers of Methanosphaera stadtmanae $\mathrm{DSMZ3091^{ \textrm {T } }}$ cross reactive $\mathrm{IgG}$ antibodies, and the archaeon was shown to be more immunogenic than Methanobrevibacter smithii in a murine model of respiratory disease $[19,20]$. In summation, we propose that the genus Methanosphaera remains underexplored and poorly defined relative to their evolutionary origins, ubiquity and abundance in the gastrointestinal tracts of warm-blooded animals, and thereby, so too are their contributions to gut function, health and disease.

Here we describe the isolation and genomic characteristics of two new isolates of Methanosphaera sp., from the bovine rumen (BMS, the first reported isolate of its type) and human stool (PA5). Based on these initial findings, we produced seven Methanosphaera spp. population genomes from human, ovine and bovine metagenomic datasets and undertook the first, pan-genomic analysis of this genus.

\section{Materials and methods}

\section{Enrichment and isolation of Methanosphaera sp. BMS and PA5}

Rumen fluid was collected using previously described procedures [17] from a fistulated Brahman steer grazing on native grass/legume forage at the Gatton campus of the University of Queensland's School of Agriculture and Food Sciences. The procedures were approved by the Department of Employment, Economic Development and Innovation (DEEDI; AEC Proposal Reference Number SA 2011/08/ $365)$. A subsample of the strained liquid $(\sim 2 \mathrm{ml})$ was mixed with an equal volume of pre-reduced, anoxic, and sterilized solution of $30 \%$ (vol/vol) glycerol, and then stored $-80{ }^{\circ} \mathrm{C}$. An inoculum was also prepared from the stool samples of a healthy adult Australian subject, recruited as part of a larger nutritional study conducted through Monash University under MU-HREC approval CF14/2904 - 2014001593 and UQ-HREC 2015000317. The raw stool samples were subsampled $(\sim 2 \mathrm{~g})$ under anaerobic conditions and mixed with $3 \mathrm{ml}$ of sterile glycerol preservation medium as described above.

Enrichment cultures of digesta and stool samples were initiated with BRN-RF30 medium [21] supplemented with either a combination of methanol and ethanol (each $1 \%$ $\mathrm{vol} / \mathrm{vol}$ ), or the same concentration of methanol but with $\mathrm{H}_{2}$ gas added to a final pressure of $150 \mathrm{kPa}$. The enrichments were placed within a shaking incubator cabinet at $37^{\circ} \mathrm{C}$ and agitated at 100 r.p.m. After 2-7 days incubation, $2 \mathrm{~mL}$ of the headspace gases were collected and analyzed for methane gas formation; and culture fluids were examined by UV fluorescence-microscopy for autofluorescent cells. The bovine-derived methanol: $\mathrm{H}_{2}$ enrichment cultures that were positive for both criteria were then used to inoculate BRN-RF30 medium supplemented with methanol: $\mathrm{H}_{2}$, and $50 \mu \mathrm{g} / \mathrm{mL}$ penicillin, with subsequent rounds of enrichment using the same basal medium with either $100 \mu \mathrm{g} / \mathrm{mL}$ penicillin, $50 \mu \mathrm{g} / \mathrm{mL}$ tetracycline, or $100 \mu \mathrm{g} / \mathrm{mL}$ novobiocin added. For the human stool-derived enrichment cultures, both ampicillin $(500 \mu \mathrm{g} / \mathrm{mL})$ and streptomycin $(80 \mu \mathrm{g} / \mathrm{mL})$ were added to the initial transfers and vancomycin $(50 \mu \mathrm{g} / \mathrm{mL})$ was also included to further suppress bacterial growth. For each round of enrichment, the inoculum was first diluted to extinction, and the culture inoculated with the greatest dilution that still produced autofluorescent cells and methane in the headspace gas used for the next round. The candidate axenic cultures were then serially diluted and used to inoculate BRN-RF30 agar roll tubes supplemented with methanol and $\mathrm{H}_{2}$ gas. Following growth, individual colonies were aseptically picked and propagated using the same medium. One isolate from each enrichment (strain BMS from bovine and PA5 from human stool) were selected for preservation based on microscopy and headspace gas analysis, and then archaea-targeting primers (86F/1340R, [22]) were used for 16S rRNA gene sequencing to confirm strain purity and their affiliation with the Methanosphaera genus, following methods described by Hoedt et al. [17]. 


\section{Methanosphaera spp. substrate utilization and growth kinetics}

Substrate affinities for $\mathrm{H}_{2}$ and/or ethanol, and maximal growth rates for Methanosphaera sp. BMS, WGK6 and DSMZ3091 ${ }^{\mathrm{T}}$ were determined using BRN-RF30 medium, with the headspace purged with oxygen-free $\mathrm{N}_{2}$ gas to remove any carryover $\mathrm{H}_{2}$, and the methods outlined by $[17,23]$. Briefly, triplicate cultures consisting of $10 \mathrm{~mL}$ BRN-RF30 in Balch tubes of each strain were provided with $1 \%$ (vol/vol) methanol and either varying concentrations of ethanol $(0,3,10,30,50$, and $171 \mathrm{mM}$ with strain WGK6) or $\mathrm{H}_{2}(0,1.3,1.8,2.6,3.5$, and $4.6 \mathrm{mM}$ for all three strains) pressurized to $150 \mathrm{kPa}$. The cultures were placed within a shaking incubator cabinet at $37^{\circ} \mathrm{C}$ and agitated at 100 r.p.m. Growth was monitored by longitudinal measurement of optical density at $600 \mathrm{~nm}\left(\mathrm{OD}_{600}\right)$ as described by Hoedt et al. [17]. Strains DSMZ3091 ${ }^{\mathrm{T}}$ (human) and BMS (bovine) were also cultured using BRN basal medium prepared to contain either human fecal water (FW) or bovine rumen fluid (RF) at different concentrations. The FW extract was prepared by resuspending $10 \mathrm{~g}$ of human fecal material in $100 \mathrm{~mL}$ of $\mathrm{ddH}_{2} \mathrm{O}$, followed by two rounds of centrifugation $\left(20,000 \times g\right.$ for $30 \mathrm{~min}$ at $\left.4{ }^{\circ} \mathrm{C}\right)$ and filter sterilization of the supernatant $(0.45 \mu \mathrm{m}$ Thermo Scientific Nalgene Rapid-Flow Filter unit). The basal BRN medium was then supplemented with either 1 or $5 \%$ (vol/vol) FW, or 5 and $10 \% \mathrm{RF}$ (vol/vol) prior to autoclaving. Triplicate tubes of the culture media were inoculated with actively growing cultures of the respective strains, the tubes aseptically pressurized with $\mathrm{H}_{2}: \mathrm{CO}_{2}(80: 20 \mathrm{vol} / \mathrm{vol})$ to $150 \mathrm{kPa}$, and $1 \%$ (vol/vol) methanol added. The cultures were placed within a shaking incubator cabinet at $37^{\circ} \mathrm{C}$ and agitated at 100 r.p.m. Growth was monitored by longitudinal measurement of optical density at $600 \mathrm{~nm}\left(\mathrm{OD}_{600}\right)$ as described above.

Specific growth rates for each substrate concentration and combination were calculated and plotted as doublereciprocal plots, with substrate affinity and maximal growth rate estimated from the $x$ - and $y$-intercepts, respectively. GraphPad Prism 7 was used to plot the optical densities over time and determine the specific growth rate using linear-regression of the exponential growth phase for each condition. Unpaired Student's $t$-test was used for the statistical analysis, and $P$-values $<0.05$ were determined to be statistically significant. Growth rates and statics were calculated as described above.

\section{Methanosphaera spp. genome sequencing}

The methods and sequencing platform used to produce the draft genome of strain WGK6 were described by Hoedt et al. [17]. Strains BMS and PA5 were cultured using BRN-RF30 medium supplemented with methanol and hydrogen and harvested by centrifugation $(17,000 \times g)$. The cell pellets were resuspended in $\mathrm{RBB}+\mathrm{C}$ lysis buffer [24] and subjected to 15 rounds of freeze-thaw cycles and repeated phenol: chloroform extraction, with a final additional ethanol precipitation included. The genomic DNA was quantified using the Quant-iT dsDNA BR Assay Kit according to the manufacturer's instructions (Invitrogen) and its integrity determined by agarose gel electrophoresis. Aliquots $(20 \mu \mathrm{g})$ of BMS genomic DNA were then sheared with the Hydroshear $^{\circledR}$ Shearing Device (Pacific Biosciences, CA, USA) to produce fragments $\sim 20 \mathrm{~Kb}$ in length, which were then used for library construction and SMRT-cell sequencing with the PacBio RS2 "continuous long read" platform and the SMRT portal assembly (Pacific Biosciences). The genome assembly was performed using HGAP2 with a seed cutoff length of $10 \mathrm{~Kb}$, and quality assessed via adjacency graph construction and visualization with Contguity [25]. Finally, genome closure was achieved using primers (Table S1) to "walk" across the determined ends of the single BMS contig, and the amplicons sequenced using an Applied Biosystems 3130xl Genetic Analyser. For strain PA5, a high-quality draft genome was prepared from $100 \mathrm{ng}$ genomic DNA by the Australian Centre for Ecogenomics (ACE, www. ecogenomic.org) using their in-house workflows for the Illumina NextSeq platform. The raw data files were quality checked, filtered, and assembled using the CLC genomics workbench 10 (www.qiagenbioinformatics.com).

The quality of the genome assemblies for strains BMS and PA5 were assessed using CheckM [26]. The BMS genome has been deposited at JGI IMG/ER under the accession 2626541600, and DDBJ/EMBL/GenBank wholegenome submission portal under the accession CP014213. The PA5 genome has been deposited under the JGI IMG/ ER accession 2754412591 and DDBJ/EMBL/GenBank accession NGJK00000000.

\section{Comparative analyses of the Methanosphaera sp. isolate genomes}

We first attempted to improve the assignment of functions to "unknown" genes, by using RAST, Prokka, NCBI, KEGG, and JGI IMG/ER annotation tools [27-30]. Additionally, the COG gene profiles, mobile genetic elements (MGE), CRISPR-Cas functions and fused protein coding genes for Methanosphaera sp. DSMZ3091 ${ }^{\mathrm{T}}$, BMS, PA5, and WGK6 genomes were produced via JGI IMG/ER. The Methanosphaera stadtmanae DSMZ3091 ${ }^{\mathrm{T}}$, BMS, WGK6, PA5, DSMZ4103 ${ }^{\mathrm{T}}$ genomes and the population genomes were uploaded to the software platform EDGAR [31] to determine a core, shared and accessory genome. The Methanosphaera DSMZ3091 ${ }^{\mathrm{T}}$, WGK6 and BMS genomes were also compared using barcodeByMers (https://github. 
com/minillinim/mikezbioscripts) across $2 \mathrm{~Kb}$ windows of each genome, and the resulting heat map was generated in RStudio (www.rstudio.com) using the package ggplot2 (http://ggplot2.org/).

\section{Retrieval of Methanosphaera population genomes from metagenomic data}

The NCBI Sequence Read Archives (SRA) were queried with the 16S rRNA from Methanosphaera stadtmanae DSMZ3091 ${ }^{\mathrm{T}}$ to identify datasets most likely to contain sequences associated with Methanosphaera spp. (Table S2). Metagenomic datasets identified as possessing Methanosphaera spp. associated sequences included 10 stool samples from the study by Karlsson et al. [32] of elderly European women with glucose control profiles considered either normal, impaired, or diabetic; and the ovine rumen metagenomic datasets from Shi et al. [16]. Bovine rumen metagenomic datasets (Illumina HiSeq 2000 platform; Macrogen Inc., Korea) produced from 10 Brahman steers consuming Rhodes grass (Chloris gayana) with or without supplementation of Leucaena (Leucaena leucocephala) as part of an unpublished CSIRO-led study were also used. The datasets were quality checked with Trimmomatic v0.32 [33] to remove adapters and trim low-quality bases, and bbmerge v34.49 (http://sourceforge.net/projects/bbmap/) was used to merge overlapping pairs. The quality checked, paired-end and merged reads were assembled using CLC Genomics Workbench 8 (http://www.clcbio.com), and MetaBAT v0.25.4 [34] was used to recover population genomes from the three metagenomic studies. An estimate of population genome completeness and contamination was performed with CheckM v1.0.3, and their estimated size was calculated from the product of bin size (in nucleotides) and CheckM completeness score [26]. Annotations for the 7 population genomes were generated through the Rapid Annotation using Subsystems Technology (RAST) server and Prokka.

The population genomes have been deposited at DDBJ/ ENA/GenBank under the following accession numbers MUZW00000000, MVJJ00000000, MVJK00000000, MVAA00000000, MUZZ00000000, MUZY00000000, and MUZX00000000. The versions described in this paper is version XXXX01000000.

\section{Genome-based nucleotide identity and phylogenetic analyses}

Mauve [35] was used to examine genome synteny among the 12 isolate and population genomes, with Methanosphaera stadtmanae DSMZ3091 ${ }^{\mathrm{T}}$ used as the reference genome. The phylogenetic trees of the amino acid sequences for methyl-coenzyme $\mathrm{M}$ reductase component $\mathrm{A} 2$
(MrtA) and energy conserving hydrogenase (EhaRST) were constructed with MEGA7 [36]. ClustalW [37]) was first used to align the sequences, and the stability of the Jones-Taylor-Thornton modelled Maximum-likelihood tree was evaluated by 1000 bootstrap replications.

The average nucleotide identity (ANI) scores between the orthologs present in a "core genome" of 293 genes from all 12 Methanosphaera genomes were also calculated using EDGAR, and a matrix of ANI scores generated using RStudio. The carbohydrate-active enzyme (CAZYme) profiles were produced using the dbCAN database (http://csbl. bmb.uga.edu/dbCAN/). Whole-genome based phylogenies were produced using either the Genome Trees Database (GTDB) which uses the concatenation of 122 universal marker genes to perform the analysis [38] or the "core genome" of 293 genes common to all 12 Methanosphaera genomes, using EDGAR [31]. The GTDB tree inference was performed with FastTree v2.1.7 [39] and included all genomes in IMG v4.510 [29] with the resulting tree imaged using ARB [40]. Finally, the number of core, non-core and singleton genes for the two genome groups were estimated using only the isolate genomes and those population genomes with CheckM scores $>90 \%(n=8)$.

\section{Results}

\section{Methanosphaera sp. BMS and PA5 are obligate hydrogen-dependent methylotrophs}

Our enrichment protocols from bovine rumen digesta and human stool produced axenic cultures of methanol dependant methanogenic prokaryotes. Amplicons of the $16 \mathrm{~S}$ rRNA gene of these isolates were only produced by archaeal-targeting primers $(86 \mathrm{~F} / 1340 \mathrm{R})$ and were found to be $97-99 \%$ identical with the 16S rRNA gene sequence of Methanosphaera stadtmanae DSMZ3091 ${ }^{\mathrm{T}}$. Based on these results, we concluded that these isolates were members of the genus Methanosphaera, and referred to as Methanosphaera sp. BMS and PA5, respectively. Growth and methane production for BMS was supported by methanol and $\mathrm{H}_{2}$, but not methylated amines, acetate, formate, propanol or a mixture of $\mathrm{CO}_{2}: \mathrm{H}_{2}$; nor could strain BMS use ethanol as an alternative to $\mathrm{H}_{2}$ to support methanogenesis. Based on the PA5 genome analysis and its extensive synteny and ANI scores with strain DSMZ3091 ${ }^{\mathrm{T}}$, as well as the absence of genes encoding dehydrogenases for $\mathrm{CO}_{2}$ or ethanol utilization (see below) we conclude that PA5 is not capable of autotrophic growth, nor utilization of ethanol as a carbon source or source of reductant, like that reported for Methanosphaera sp. WGK6 [17]. For these reasons, we only compared the growth of BMS with isolates from other hosts, i.e., WGK6 [17] and DSMZ3091 ${ }^{\mathrm{T}}$ using methanol as 
carbon source and varying concentrations of $\mathrm{H}_{2}$ or ethanol as the reductant. The specific growth rates of both DSMZ3091 ${ }^{\mathrm{T}}$ and WGK6 were found to be maximal with the lower concentrations of $\mathrm{H}_{2}$ added, whereas the growth rate of strain BMS was significantly increased at higher $\mathrm{H}_{2}$ concentrations (supplementary Table S3). The $\left[K_{S}\right]$ values for $\mathrm{H}_{2}$ were calculated to be $0.09 \mathrm{mM}, 0.11 \mathrm{mM}$, and 1.58 $\mathrm{mM}$ for strains WGK6, DSMZ3091 ${ }^{\mathrm{T}}$, and BMS, respectively. As expected, only strain WGK6 was capable of growth with methanol:ethanol and interestingly, its growth rate was slower with a high concentration of $\mathrm{H}_{2}$ compared to when ethanol was provided (Fig. 1 and Table S3). Based on these results, we conclude that all three strains from the different animal hosts are capable of growth with relatively low $\mathrm{H}_{2}$ thresholds, but the isolates recovered from monogastric hosts are perhaps better adapted to these conditions, and in the case of strain WGK6, have acquired accessory functions in support of methanol reduction, methanogenesis and growth.

\section{The Methanosphaera sp. BMS genome is remarkable in terms of its size}

The Methanosphaera sp. BMS genome was assembled into a single molecule with 60x coverage using the PacBio RS2 platform, and is comprised of $2,874,170 \mathrm{bp}$, nearly twice the size of the genomes of the Methanosphaera isolates derived from monogastric hosts (Table 1). The MAUVE alignments validated there is a large amount of genomic synteny among
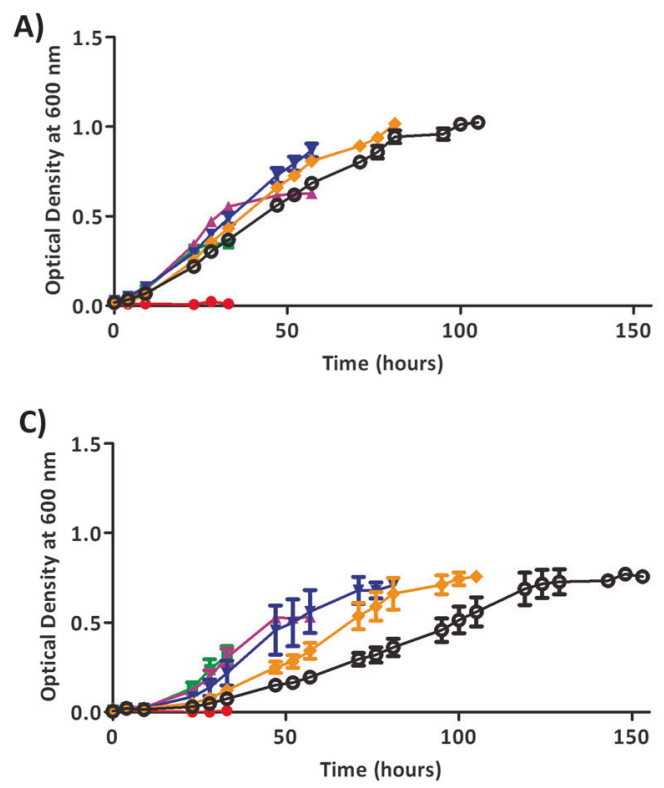

Fig. 1 Longitudinal monitoring of the growth of Methanosphaera stadtmanae DSMZ3091 ${ }^{\mathrm{T}}(\mathbf{a})$, strain BMS (b) and strain WGK6 when provided with either methanol: $\mathrm{H}_{2}$ (c) or methanol:ethanol (d). The colored lines and symbols denote different concentrations of either $\mathrm{H}_{2}$ $(0 \bullet, 1.3 \square, 1.8 \wedge, 2.6 \nabla, 3.5 \diamond$ and $4.6 \mathrm{mM})$ or in the instance of the isolates, with the "additional" content within the BMS genome demarcated from the smaller genomes via a contiguous region of $\sim 1 \mathrm{Mbp}$ (Fig. 2). Next, both CheckM and BarcodeByMers were used to assess whether the BMS genome might be comprised of a chimeric assembly, but the contamination estimates (Table 1 and Table S4), together with the uniformity in tetramer nucleotide profiles for the BMS, WGK6 and DSMZ3091 ${ }^{\mathrm{T}}$ genomes (Figure S1) suggest the differences in genome size are not the result of a chimeric assembly nor other sequencing artefacts.

The general genome features as well as the COG based assignments for the DSMZ3091 ${ }^{\mathrm{T}}$, PA5, WGK6, and BMS genomes are shown in Tables S5 and S6, respectively. In general terms, the total gene count in most COG categories are similar for all three genomes despite their different sizes, further establishing the relatedness among the strains with respect to core functions. However, only $37 \%$ of the additional gene content from the BMS genome could be assigned to functional $\mathrm{COG}$ categories such as signal transduction mechanisms (8 genes), secondary metabolite biosynthesis, transport and catabolism (10 genes), or replication, recombination and repair (16 genes). Instead, the additional genome content was predominantly comprised of either CDSs with no assigned COG functions (976 genes for strain BMS c.f. 576, 525 and 534 genes for strains WGK6, DSMZ3091 ${ }^{\mathrm{T}}$ and PA5, respectively) or COGs with "function unknown" (105 genes for strain BMS c.f. 78, and 78, and 81 genes for strains WGK6, and DSMZ3091 ${ }^{\mathrm{T}}$, and PA5, respectively). The BMS genome was also remarkable

B)

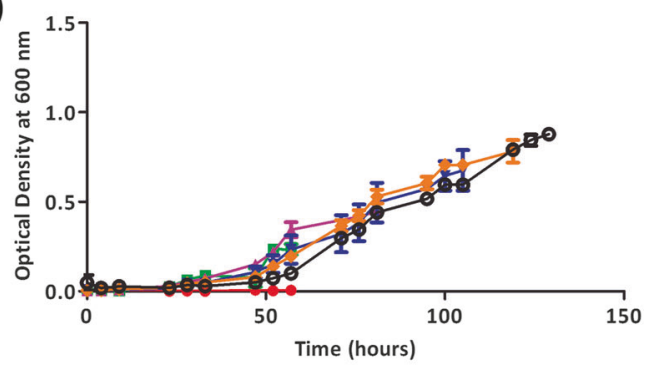

D)

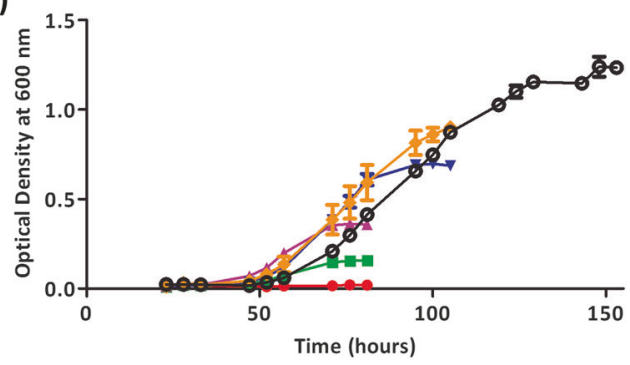

(d), ethanol $(0 \bullet, 3 \square, 10 \Delta, 30 \nabla, 50 \diamond$ and $171 \mathrm{mM})$. The growth of the individual cultures is plotted until the time point at which maximal $\mathrm{OD}_{600}$ was measured. Individual values represent the mean $( \pm \mathrm{SD})$ produced from triplicate cultures 
Table 1 Summary statistics of the Methanosphaera isolate and population genomes, with respect to population genome size, completeness and contamination estimates, and the predicted complete genome size, based on these metrics

\begin{tabular}{|c|c|c|c|c|c|c|c|c|}
\hline $\begin{array}{l}\text { Genome } \\
\text { designation }\end{array}$ & Source & Location & $\begin{array}{l}\text { Genome } \\
\text { size (bp) }\end{array}$ & GC (\%) & $\begin{array}{l}\text { CheckM } \\
\text { completeness } \\
(\%)\end{array}$ & $\begin{array}{l}\text { CheckM } \\
\text { contamination } \\
(\%)\end{array}$ & $\begin{array}{l}\text { Predicted } \\
\text { complete } \\
\text { genome size } \\
(\mathrm{Mbp})\end{array}$ & $\begin{array}{l}\text { NCBI accession } \\
\text { number }\end{array}$ \\
\hline
\end{tabular}

\begin{tabular}{|c|c|c|c|c|c|c|c|c|}
\hline \multicolumn{9}{|l|}{ Axenic cultures } \\
\hline${ }^{D S M Z 3091} 1^{\mathrm{T}}$ & Human & US & $1,767,403$ & 28.0 & 97.6 & 0 & 1.8 & NC007681 \\
\hline WGK6 & Kangaroo & AUS & $1,729,155$ & 28.0 & 96.8 & 0 & 1.7 & JRWK01000000 \\
\hline PA5 & Human & AUS & $1,701,735$ & 27.6 & 97.6 & 0 & 1.7 & NGJK00000000 \\
\hline BMS & Bovine & AUS & $2,874,170$ & 33.0 & 96.8 & 0.8 & 2.9 & СР014213 \\
\hline DSMZ4103 ${ }^{\mathrm{T}}$ & Rabbit & $\begin{array}{l}\text { Italy } \\
\text { (NZ/USA } \\
\text { breeds) }\end{array}$ & $1,928,201$ & 28.0 & 97.6 & 0 & 1.9 & LMVN00000000 \\
\hline \multicolumn{9}{|c|}{ Population Genomes } \\
\hline DEW79 & Human & Europe & $1,594,581$ & 27.6 & 97.2 & 0.8 & 1.6 & MUZW00000000 \\
\hline SHI613 & Ovine & $\mathrm{NZ}$ & $1,859,301$ & 33.0 & 90.4 & 0 & 2.1 & MVJJ00000000 \\
\hline SHI1033 & Ovine & NZ & $1,496,386$ & 29.9 & 74.3 & 0 & 2.0 & MVJK00000000 \\
\hline rholeuAM6 & Bovine & AUS & $1,935,828$ & 32.9 & 92.7 & 0 & 2.1 & MVAA00000000 \\
\hline rholeuAM74 & Bovine & AUS & $2,004,016$ & 35.5 & 75.9 & 0.8 & 2.6 & MUZZ00000000 \\
\hline rholeuAM130 & Bovine & AUS & $2,057,527$ & 34.8 & 77.6 & 4.7 & 2.5 & MUZY00000000 \\
\hline rholeuAM270 & Bovine & AUS & $1,763,900$ & 35.5 & 61.8 & 0 & 2.9 & MUZX00000000 \\
\hline
\end{tabular}
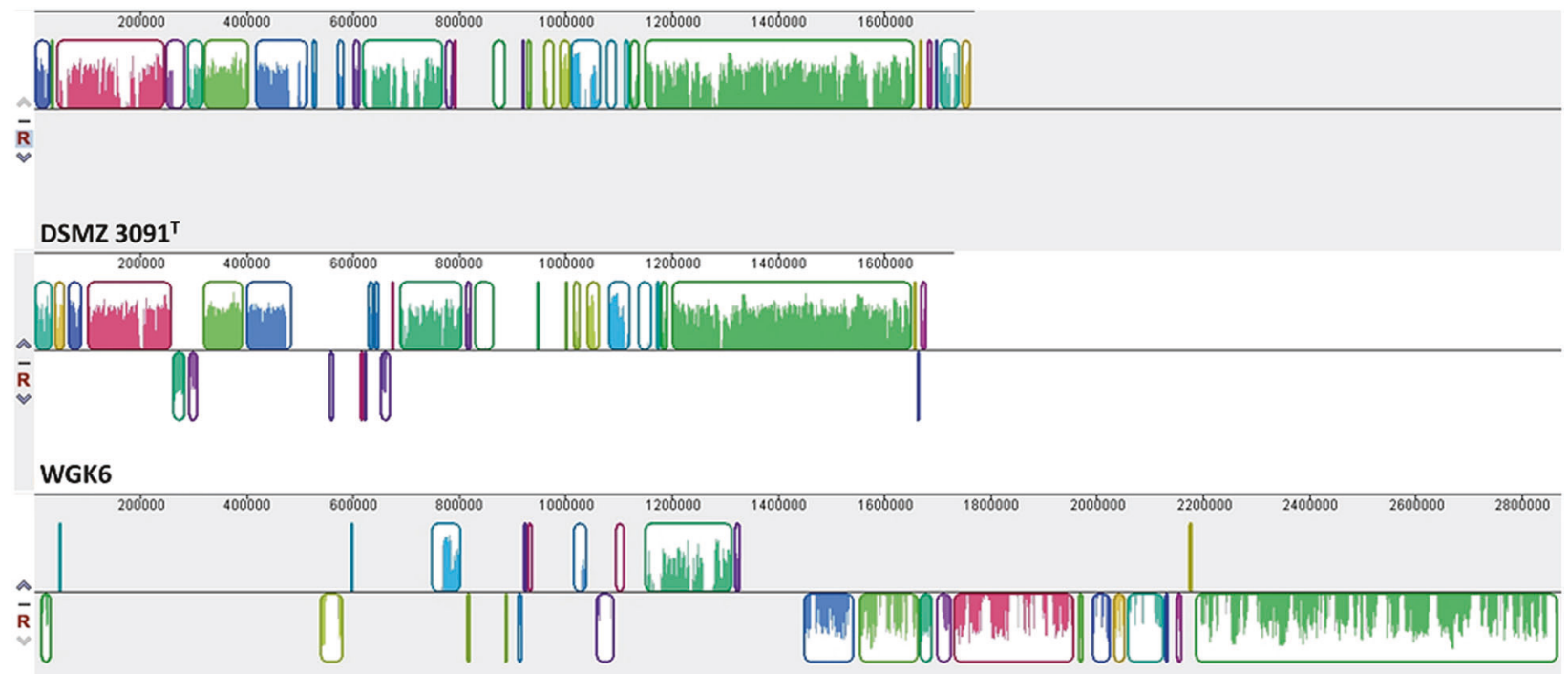

BMS

Fig. 2 The degree of genome synteny evident between the Methanosphaera stadtmanae DSMZ3091 ${ }^{\mathrm{T}}$, WGK6, and BMS genomes using Mauve. In brief detail, the alignment algorithm facilitates the depiction of genome synteny (illustrated by the size and the coloring scheme used for the local collinear blocks identified by Mauve), regions of

for its greater number of mobile genetic elements (MGE), with only a single gene annotated as a transposase in the DSMZ3091 $^{\mathrm{T}}$ genome and none in WGK6, compared to 30 putative transposases in the BMS genome. Our comparisons of the CRISPR-Cas associated proteins is limited only to those isolates available for JGI IMG/ER analysis genome rearrangement (illustrated via the lines connecting locally collinear blocks between the two genome models) and any xenologous regions (illustrated by blank spaces). The xenologous regions present in the BMS genome are not ubiquitous, as illustrated by the high degree of synteny between all three genomes

(Table S7). The PA5 genome contains no Cas annotations, while the BMS genome is predicted to possess only a duplicated copy of Cas1. In contrast, both the WGK6 and DSMZ3091 $^{\mathrm{T}}$ genomes possess CRISPR-associated proteins Cas1-7. As such, it would appear the extra genome content in strain BMS does not include a more expansive CRISPR- 
Cas system. Based on these findings, we concluded that the bovine-derived isolate BMS is a member of the Methanosphaera genus, and it is the first isolate of its kind-and distinct from the human and kangaroo isolates-because of its substantially larger genome, which is comprised of largely cryptic functions.

\section{Methanosphaera sp. population genome recovery and analyses expand the representation of the genus and confirms its divergence relative to genome size}

We were able to retrieve seven Methanosphaera spp. population genomes from two publicly available metagenomic datasets and an ongoing CSIRO project, and their predicted genome sizes are estimated from the completeness and contamination scores calculated using CheckM. They range in size from 1.6 (DEW79) to 2.7 Mbp (rholeuAM74) and $5 / 7$ are closer in size to the BMS genome described above (Table 1). Despite some of the population genomes falling below the $90 \%$ completeness threshold for core genome calculations, the extent of sequence conservation across all 12 Methanosphaera genomes is indeed extensive. First, we subjected the "large" and "small" genomes to Mauve alignments separately, which confirmed there is a high level of synteny between the sequenced isolate genomes and the bioinformatically recovered population genomes assigned to each group (Figure S2 and S3). Importantly, the Mauve alignments also confirmed the existence of syntenic blocks extending across the BMS 1 Mbp "unique" region present in the larger genomes. Next, we specifically examined the hydrogenase gene operon (EhaRST), which is universally present in all 12 genomes, and its flanking regions (Figure S4). Here, the degree of gene conservation and organization is again extensive and remarkable. Furthermore, the carbohydrate-active enzyme (CAZymes) family profiles of the 12 Methanosphaera genomes are all very similar, suggesting their evolutionary conservation and provision of key housekeeping functions (Table S8). As might be expected, there is a relatively scant representation of glycoside hydrolases $(\mathrm{GH})$; with the notable exception of all genomes containing a single GH109, which would encode a presumptive $\alpha-\mathrm{N}$ acetylgalactosaminidase [41, 42]. Conversely, both the carbohydrate esterase (CE) and glycosyltransferases (GT) families were numerically more abundant across all 12 genomes, and in particular the GT2 and GT4 families, most likely involved with surface protein glycosylation [43, 44]. Interestingly, the dbCAN analysis predicts that all the genomes contain a similar number of CAZymes with "auxiliary activities" (i.e., AA3 and AA6), which in other microbes are presumptive 1,4-benzoquinone reductases (E. C. 1.6.5.6) and involved with the conversion of NADPH,
$\mathrm{H}^{+}$and p-benzoquinone to $\mathrm{NADP}^{+}$and hydroquinone. The presence of a putative benzoquinone interacting enzyme within organisms known to have methanophenazine instead of quinones was unexpected [12]. Both KEGG Koala and NCBI BLAST searches did not identify other genes encoding "ubiquinone and other terpenoid-quinone biosynthesis". Taken together, while these AA3- and AA6containing genes appear to be conserved across the current representatives of the Methanosphaera genus, their respective functions remain cryptic. Finally, we produced a "core genome" from all 12 Methanosphaera genomes, which is comprised of 293 genes (Table S9) and used these to construct the ANI matrix among the 12 genomes (Figure S5). The ANI scores among DSMZ3091 ${ }^{\mathrm{T}}$, PA5, and DEW79 exceed 95\%, which is the generally accepted threshold for species-level grouping [45], and across all 12 genomes, range between 77 and $85 \%$, exceeding the threshold for genus-level grouping [46]. Collectively, these results show that the population genomes are truly derived from Methanosphaera sp. present within the sampled microbial communities and that representatives with large genomes a more numerous than currently appreciated, but appear to be largely restricted to ruminant animals.

\section{Phylogenetic analyses establish the monophyletic origin and a host-specific separation of Methanosphaera spp. with large genomes}

We recovered all Methanosphaera-affiliated 16S rRNA gene sequences from NCBI database for phylogenetic comparison and found there was a clear bifurcation of the available sequences between monogastric and ruminant hosts (Fig. 3). In relative terms, the BMS 16S rRNA gene represents a deep branch within the ruminant-derived clones, especially in comparison to the 16S rRNA genes from those isolates with a smaller genome (i.e., Methanosphaera stadtmanae DSMZ3091 ${ }^{\mathrm{T}}$, Methanosphaera cunculii DSMZ4103 ${ }^{\mathrm{T}}$, and Methanosphaera spp. WGK6, and PA5), which all cluster with other sequences derived from monogastric gut microbiomes. The phylogenetic trees produced from BLASTp alignments of the methylcoenzyme $\mathrm{M}$ reductase component A2 (MrtA) and the energy conserving hydrogenase (EhaR) coding sequences also confirm their monophyletic origin and separation on the basis of genome size (Figures S6 and S7) and recapitulates the phylogenetic tree produced using the GTDB universal marker gene approach (Figure S8). Interestingly though, the phylogenetic tree produced using EDGAR, from the 293 genes shared by all 12 Methanosphaera spp. genomes, results in all the representatives with a small genome being clustered together as a distinct lineage relative to those representatives with large genomes (Fig. 4a). Taken together, these results suggest that the 


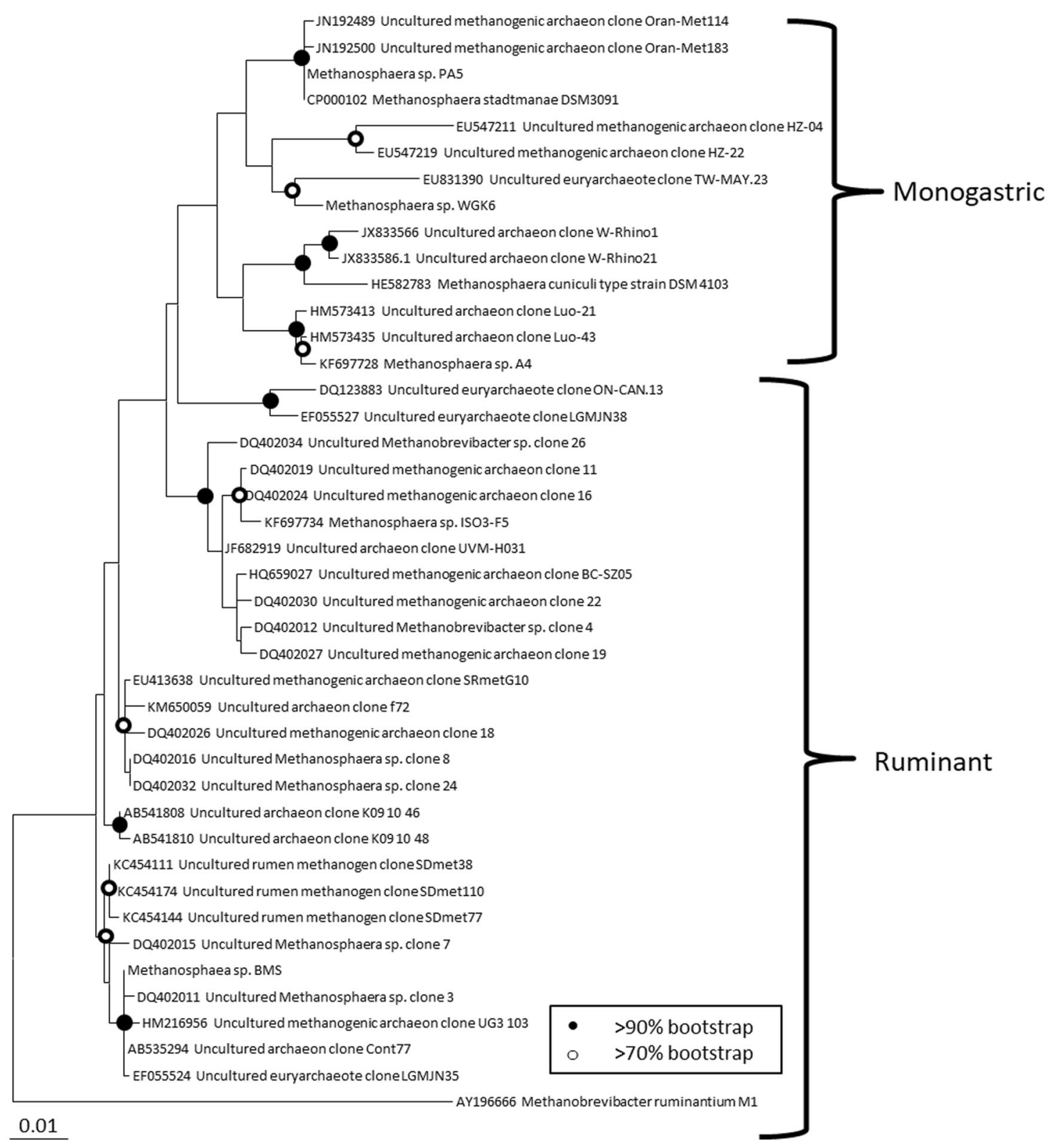

Fig. 3 Maximum-likelihood phylogenetic analysis of Methanosphaera spp. isolate and $r r s$ gene clone sequences recovered from NCBI. There is a clear separation of the available Methanosphaera spp. rrs

Methanosphaera genus is monophyletic in origin and those representatives with large genomes represent more ancestral lineages that are principally adapted to ruminant hosts.

\section{The differences in growth of Methanosphaera isolates BMS and DSMZ3091 ${ }^{\top}$ using habitat simulating medium suggests host-specificity}

To biologically assess the apparent differences in host specificity predicted by the phylogenetic analyses described above, extracts prepared from either bovine rumen fluid (RF) or human faecal water (FW) were added to the basal sequences between monogastric and ruminant hosts. Bootstrap values are shown and the scale bar represents $1 \%$ sequence divergence, with Methanobrevibacter ruminantium M1 used as the outgroup

culture medium to produce a bovine and human habitatsimulating medium, respectively. The results of these growth studies are shown in Fig. 5 and Table S10. Both the growth rate and final yield of the human isolate DSMZ3091 $^{\mathrm{T}}$ was greater than that observed for the bovine strain BMS and was similar in both RF and FW containing media, irrespective of the amount added. Conversely, the growth kinetics for strain BMS were maximal in RFcontaining media and substantially decreased with FW containing medium, in a concentration-dependent manner. These results suggest that the composition of RF and FW have limited effects on the growth kinetics of the human 
A)

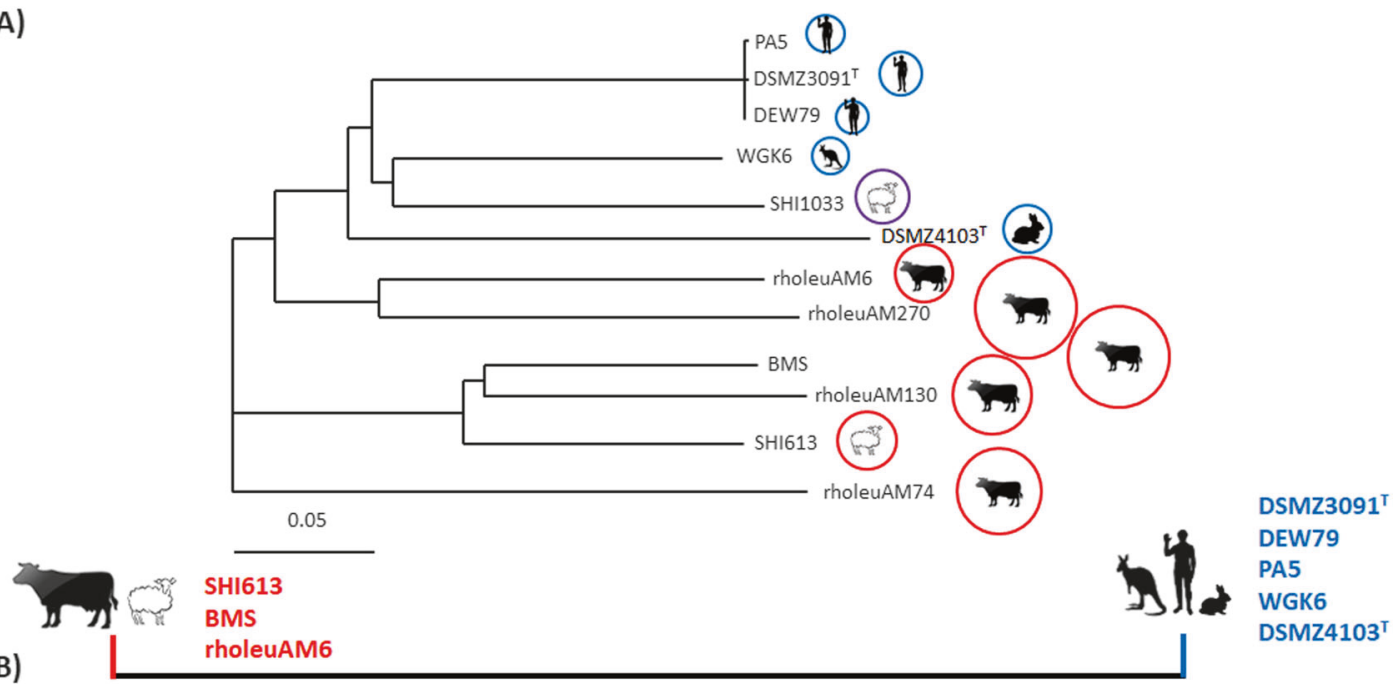

B)

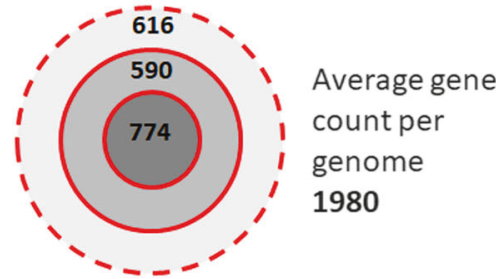

Fig. 4 a The genome based phylogenetic tree of the isolate and population genomes recovered for Methanosphaera spp. inferred from the concatenation of 293 genes from the core genome and using EDGAR. Those representatives with "small" genomes (<1.8 Mbp; blue/purple circles) appear to have evolved more recently and were more readily found in monogastric hosts, whereas representatives with "large" genomes ( $>2.0 \mathrm{Mbp}$; red circles) appear restricted to ruminant hosts and the deeper branching suggestive of their ancestral age.

isolate, whereas the bovine isolate (BMS) requires growth factors which are either absent or reduced in FW compared to RF.

\section{Discussion}

Here we have expanded the genomic representation of the genus Methanosphaera from 3 to 12 members, with the addition of two new axenic isolates of ruminant and human origin, as well as the reconstruction of seven population genomes from both human and ruminant metagenomic datasets. Despite being a well-recognized member of the Methanobacteriales with niche specificity first confirmed in human subjects and subsequently, the gastrointestinal tracts of other warm-blooded animals, the physiological capacity of Methanosphaera spp. has been for the most part overlooked. Methanosphaera spp. have been reported to be enriched in "low methane" emitting ruminants [16], with at least some isolates employing alcohol-fueled methanogenesis [17].

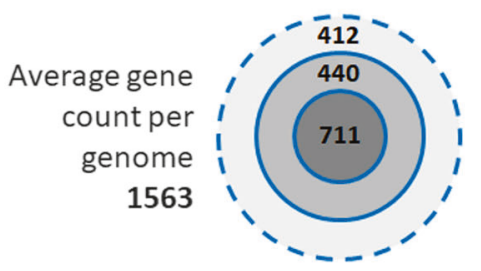

b Estimates of the core, non-core, and singleton genes present in the isolate and population genomes with $>90 \%$ completeness, when expressed as a per genome equivalent. The total number of core genes is relatively stable between the "large" (red) and "small" (blue) genome groups, with the key differences between groups represented by the substantially larger counts of predicted non-core and singleton genes

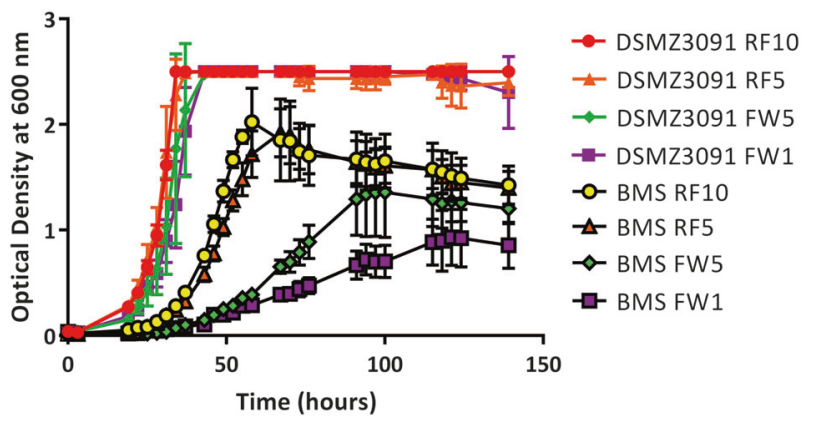

Fig. 5 Growth of human Methanosphaera stadtmanae DSMZ3091 ${ }^{\mathrm{T}}$ and bovine Methanosphaera sp. BMS when cultured using BRN basal medium supplemented with either 5 or $10 \%$ (vol/vol) rumen fluid (RF5 and RF10, respectively) or 1 and $5 \%$ (vol/vol) human faecal water (FW1 and FW5, respectively). Notably, the growth rate and yield of strain DSMZ3091 ${ }^{\mathrm{T}}$ was only marginally affected by the different RF and FW concentrations; however, both the growth rate and yield of strain BMS was reduced in the presence of FW in comparison to RFcontaining cultures. Individual values represent the mean $( \pm \mathrm{SD})$ produced from triplicate cultures

Our isolation of a ruminant-derived isolate of Methanosphaera sp. (BMS) has provided the first evidence for the 
existence of representatives with different genome sizes: with a large genomotype ( 2.9 Mbp) compared to those Methanosphaera isolates from human and macropodid hosts ( 1.7 Mbp). The regions "unique" to the BMS genome were used for BLASTn analyses against the NCBI databases, and no significant matches to large blocks of other bacterial or archaeal genomes were recovered. The BarcodeByMers analysis also showed that the tetranucleotide profiles of three fully sequenced genomes from human, macropodid, and ruminant origin are virtually identical. Furthermore, the ANI scoring and the Mauve alignments of the BMS and large population genomes revealed the existence of large syntenic blocks extending across the $1 \mathrm{Mbp}$ "unique" region present in these genomes. Taken together, these results suggest that the BMS genome is not a chimeric assembly and the extra genome content is unlikely to be the result of recent gene transfer events. Based on these findings, we propose that the Methanosphaera genus is monophyletic, but actually comprised of two genomotypes. The phylogenetic trees constructed from the genomic data of our isolates and the Methanosphaera-affiliated population genomes also supports our hypothesis that the Methanosphaera spp. with a large genome represent lineages restricted to ruminant hosts (Figs. 3, 4 and supplementary Figures S6, S7 and S8). In that context, we recovered seven Methanosphaera-affiliated population genomes, and 5/7 of these are large, and all 5 were recovered from ruminant-derived datasets (Table 1). The EDGAR phylogenetic tree for all 12 Methanosphaera genomes, constructed using a concatemer of "core genes" from each representative, produced a clear separation of the Methanosphaera spp. with the smaller genomotypes (i.e., DSMZ3091 $^{\mathrm{T}}$, PA5, DEW79, WGK6, DSMZ4103 ${ }^{\mathrm{T}}$, and SHI1033) from the large genomotypes (Fig. 4).

In general terms, the total gene count in most defined COG categories are similar between the two genomotypes despite their different sizes, further establishing the relatedness among the strains with respect to core functions. An example of the extent of sequence conservation across the genus is illustrated for the energy conserving hydrogenase gene (EhaRST) and flanking regions (Figure S3). Remarkably, the carbohydrate-active enzyme (CAZy) profiles of the respective genomes, and in particular the total gene count and representation of different glycosyltransferases (GT) families appears to be strongly conserved across the two genomotypes. The GT2 and GT4 families are the most numerous across all the genomes (Table S8) and an unusual NAD-dependent $\alpha$-N-acetylgalactosaminidase (GH109) is also conserved across the Methanosphaera-affiliated genomes, along with multiple copies of GT28 genes, which are infrequently observed in Archaea, but are proposed to be a part of the minimal 3-gene set for peptidoglycan metabolism in other organisms [47]. Collectively, these findings raise new insights into cell wall biosynthesis and decoration in methanogenic archaea, both of which may play substantive roles in coordinating host-microbe interactions and immunomodulation.

Although, the pan-genome analyses revealed a conserved set of functions coordinating the hydrogen-dependent reduction of methanol to methane and emphasizes the limited nutritional versatility of the Methanosphaera genus, there were also some notable differences observed. The SHI1033 population genome was recovered from the sheep rumen metagenomic data of "low methane" emitting sheep [16] and is most similar (in size and content, Figure S9) to strain WGK6, isolated from a macropodid (kangaroo) host. Interestingly, both the WGK6 and SHI1033 genomes possess the alcohol and aldehyde dehydrogenase genes that are considered key to the hydrogen-independent growth of strain WGK6 [17]. The macropodids are recognized as being "low methane emitters" compared to ruminant livestock [48] but more recently, "low methane" emitting ruminants have been identified. These animals possess a shorter retention of feed within the rumen, and a bacterial "ruminotype" associated with reduced levels of ruminal hydrogen production [49]. As such, the capacity for alcohol-fueled methanogenesis by some Methanosphaera spp. appears to be an adaptation to survival in low hydrogen environments of "low methane emitting" animals.

Our gene- and genome-based phylogenetic analyses support the bifurcation of Methanosphaera spp. on the basis of genome size and host specificity; with strain BMS and the representatives with a large genome size recovered only from ruminant hosts. We therefore conducted growth studies with the human and bovine-derived strains of Methanosphaera using media prepared to simulate the growth habitats of the bovine rumen and human gut, by the provision of either rumen fluid or human fecal water, respectively. Interestingly, the growth kinetics of the human isolate were largely unchanged by the provision of either RF or FW at different concentrations, suggesting their growth is not ratelimited by the nutrient milieu present in both types of extracts. Conversely, while the growth kinetics of bovinederived strain BMS were essentially the same when cultured with RF added at 5 or $10 \%$ ( $\mathrm{vol} / \mathrm{vol})$, the substitution of RF with FW did result in a marked decrease in both growth rate and yield. Furthermore, the reductions in strain BMS growth kinetics observed when FW was reduced from 5 to $1 \%$ (vol/ vol) suggests the FW nutrient milieu is rate-limiting to the growth, rather than FW possessing non-nutrient factors antagonistic to BMS growth (e.g., anti-microbial proteins). Taken together, these findings suggest that the nutrient requirements of Methanosphaera spp. with larger genomes are more complex than those with smaller genomes, and is a reflection of their additional gene content. Such findings, while limited in scope, are also consistent with our inability to recover representatives of Methanosphaera spp. with 
large genomes from monogastric hosts; and the recovery of a representative of Methanosphaera spp. with a small genome from a ruminant host (SHI1033).

The evolutionary origins of the gut microbiota, particularly in humans, remain enigmatic. While progress has been made with establishing some of the major lineages of hominid gut bacteria diverged coordinately (co-speciated) with their host [50], scant information is available for methanogenic archaea, and in particular the genus Methanosphaera. Here, our combined use of cultivation- and metagenomics-methods have provided an increased representation of the Methanosphaera genus that is sufficient to reveal novel insights into their phylogeny, and that the variation in genomic content across the genus likely contributes to host-specificity. The genome-scale phylogenetic and comparative analyses confirmed the monophyletic origin of the genus Methanosphaera, which can be bifurcated into two genomotypes, with the smaller genomotype enriched in animal hosts with a "monogastric" digestive system and the large genomotype restricted to ruminant hosts.

Acknowledgements E.C.H. has been supported by an Australian Postgraduate Award. The financial support of Meat and Livestock Australia Limited via a Technical Assistance Grant (to E.C.H. project code B.STU.0257) as well as funds for project D.MHN.0617 (awarded to J.G.M. and P.R.G.) is gratefully acknowledged. This work has also been supported by funds (to M.M.) via CSIRO's Science Leader scheme and The University of Queensland Diamantina Institute. The Translational Research Institute is supported by a grant from the Australian Government. The CSIRO projects 01200.035/B.CCH.6510 and 01200.038/B.CCH.6610 were funded by the Australian Government Department of Agriculture, and Meat \& Livestock Australia Limited as part of the National Livestock Methane Program. P.Ó.C. is supported by a UQ Reginald Ferguson Research Fellowship in Gastroenterology. We are especially grateful for the technical assistance received with the PacBio sequencing of the BMS genome by Paul Lacaze in his past role with Millennium Science and Lawrie Wheeler (Queensland Institute of Technology), as well as gas chromatography analysis support by Mr Jagadish Padmanabha.

\section{Compliance with ethical standards}

Conflict of interest The authors declare that they have no conflict of interest.

Ethical approval E.C.H. planned and performed research and helped write the paper; D.H.P., J.G.V., C.P.R., C.S.M., S.E.D. and P.Ó.C. assisted with research and helped write the paper. P.R.G. and J.G.M. provided samples enabling Methanosphaera sp. PA5 isolation and helped with the preparation and review of the manuscript; and P.H., G.W.T., and M.M. planned and supervised research and wrote the paper.

\section{References}

1. Brusa T, Canzi E, Allievi L, Puppo E, Ferrari A. Methanogens in the human intestinal tract and oral cavity. Curr Microbiol. 1993;27:261-5.
2. Evans PN, Hinds LA, Sly LI, McSweeney CS, Morrison M, Wright AD. Community composition and density of methanogens in the foregut of the Tammar wallaby (Macropus eugenii). Appl Environ Microbiol. 2009;75:2598-602.

3. Mao SY, Yang CF, Zhu WY. Phylogenetic analysis of methanogens in the pig feces. Curr Microbiol. 2011;62:1386-9.

4. Borrel G, McCann A, Deane J, Neto MC, Lynch DB, Brugere J-F, et al. Genomics and metagenomics of trimethylamine-utilizing Archaea in the human gut microbiome. ISME J. 2017; 11:2059-2074.

5. Poulsen M, Schwab C, Borg Jensen B, Engberg RM, Spang A, Canibe N, et al. Methylotrophic methanogenic Thermoplasmata implicated in reduced methane emissions from bovine rumen. Nat Commun. 2013;4:1428.

6. Dridi B, Fardeau ML, Ollivier B, Raoult D, Drancourt M. Methanomassiliicoccus luminyensis gen. nov., sp. nov., a methanogenic archaeon isolated from human faeces. Int J Syst Evol Microbiol. 2012;62:1902-7.

7. Hansen EE, Lozupone CA, Rey FE, Wu M, Guruge JL, Narra A, et al. Pan-genome of the dominant human gut-associated archaeon, Methanobrevibacter smithii, studied in twins. Proc Natl Acad Sci USA. 2011;108:4599-606.

8. Leahy SC, Kelly WJ, Altermann E, Ronimus RS, Yeoman CJ, Pacheco DM, et al. The genome sequence of the rumen methanogen Methanobrevibacter ruminantium reveals new possibilities for controlling ruminant methane emissions. PLoS ONE. 2010;5: e8926.

9. Leahy SC, Kelly WJ, Li D, Li Y, Altermann E, Lambie SC, et al. The complete genome sequence of Methanobrevibacter sp. AbM4. Stand Genom Sci. 2013;8:215-27.

10. Rea S, Bowman JP, Popovski S, Pimm C, Wright A-DG. Methanobrevibacter millerae sp. nov. and Methanobrevibacter olleyae sp. nov., methanogens from the ovine and bovine rumen that can utilize formate for growth. Int J Syst Evolut Microbiol. 2007;57:450-6.

11. Miller TL, Wolin MJ. Methanosphaera stadtmaniae gen. nov., sp. nov.: a species that forms methane by reducing methanol with hydrogen. Arch Microbiol. 1985;141:116-22.

12. Thauer RK, Kaster AK, Seedorf H, Buckel W, Hedderich R. Methanogenic archaea: ecologically relevant differences in energy conservation. Nat Rev Microbiol. 2008;6:579-91.

13. Yan Z, Wang M, Ferry JG. A ferredoxin- and F420H2-dependent, electron-bifurcating, heterodisulfide reductase with homologs in the domains bacteria and archaea. MBio. 2017;8:e02285-16.

14. Gilmore SP, Henske JK, Sexton JA, Solomon KV, Seppala S, Yoo JI, et al. Genomic analysis of methanogenic archaea reveals a shift towards energy conservation. BMC Genom. 2017;18:639.

15. Biavati B, Vasta M, Ferry JG. Isolation and characterization of "Methanosphaera cuniculi" sp. nov. Appl Environ Microbiol. 1988;54:768-71.

16. Shi W, Moon CD, Leahy SC, Kang D, Froula J, Kittelmann S, et al. Methane yield phenotypes linked to differential gene expression in the sheep rumen microbiome. Genome Res. 2014;24:1517-25.

17. Hoedt EC, Cuiv PO, Evans PN, Smith WJM, McSweeney CS, Denman SE, et al. Differences down-under: alcohol-fueled methanogenesis by archaea present in Australian macropodids. ISME J. 2016;10:2376-88.

18. Blais Lecours P, Marsolais D, Cormier Y, Berberi M, Haché C, Bourdages R, et al. Increased Prevalence of Methanosphaera stadtmanae in Inflammatory Bowel Diseases. PLoS ONE. 2014;9: e87734.

19. Bernatchez E, Gold MJ, Langlois A, Blais-Lecours P, Boucher M, Duchaine $\mathrm{C}$, et al. Methanosphaera stadtmanae induces a type IV hypersensitivity response in a mouse model of airway inflammation. Physiol Rep. 2017;5:e13163 
20. Blais Lecours P, Duchaine C, Taillefer M, Tremblay C, Veillette $\mathrm{M}$, Cormier Y, et al. Immunogenic properties of archaeal species found in Bioaerosols. PLoS ONE. 2011;6:e23326.

21. Joblin KN, Naylor GE, Williams AG. Effect of Methanobrevibacter smithii on xylanolytic activity of anaerobic ruminal fungi. Appl Environ Microbiol. 1990;56:2287-95.

22. Wright AD, Pimm C. Improved strategy for presumptive identification of methanogens using $16 \mathrm{~S}$ riboprinting. J Microbiol Methods. 2003;55:337-49.

23. Kovarova-Kovar K, Egli T. Growth kinetics of suspended microbial cells: from single-substrate-controlled growth to mixedsubstrate kinetics. Microbiol Mol Biol Rev. 1998;62:646-66.

24. Yu Z, Morrison M. Improved extraction of PCR-quality community DNA from digesta and fecal samples. BioTechniques. 2004;36:808-12.

25. Sullivan MJ, Ben Zakour NL, Forde BM, Stanton-Cook M, Beatson SA. Contiguity: contig adjacency graph construction and visualisation. PeerJ Prepr. 2015;3:e1273.

26. Parks DH, Imelfort M, Skennerton CT, Hugenholtz P, Tyson GW. CheckM: assessing the quality of microbial genomes recovered from isolates, single cells, and metagenomes. Genome Res. 2015;25:1043-55.

27. Aziz RK, Bartels D, Best AA, DeJongh M, Disz T, Edwards RA, et al. The RAST Server: rapid annotations using subsystems technology. BMC Genom. 2008;9:75.

28. Kanehisa M, Goto S. KEGG: Kyoto encyclopedia of genes and genomes. Nucleic Acids Res. 2000;28:27-30.

29. Markowitz VM, Chen I-MA, Palaniappan K, Chu K, Szeto E, Grechkin Y, et al. IMG: the integrated microbial genomes database and comparative analysis system. Nucleic Acids Res. 2012;40:D115-22.

30. Seemann T. Prokka: rapid prokaryotic genome annotation. Bioinformatics. 2014;30:2068-9.

31. Blom J, Albaum SP, Doppmeier D, Puhler A, Vorholter FJ, Zakrzewski M, et al. EDGAR: a software framework for the comparative analysis of prokaryotic genomes. BMC Bioinform. 2009; 10:154.

32. Karlsson FH, Tremaroli V, Nookaew I, Bergstrom G, Behre CJ, Fagerberg B, et al. Gut metagenome in European women with normal, impaired and diabetic glucose control. Nature. 2013;498:99-103.

33. Bolger AM, Lohse M, Usadel B. Trimmomatic: a flexible trimmer for Illumina sequence data. Bioinformatics. 2014;30:2114-20.

34. Kang DD, Froula J, Egan R, Wang Z. MetaBAT, an efficient tool for accurately reconstructing single genomes from complex microbial communities. PeerJ. 2015;3:e1165.

35. Darling AE, Mau B, Perna NT. Progressive Mauve: multiple genome alignment with gene gain, loss and rearrangement. PLoS ONE. 2010;5:e11147.

36. Kumar S, Stecher G, Tamura K. MEGA7: Molecular Evolutionary Genetics Analysis Version 7.0 for Bigger Datasets. Molecular Biology and Evolution. 2017;33:1870-1874
37. Thompson JD, Higgins DG, Gibson TJ. CLUSTAL W: improving the sensitivity of progressive multiple sequence alignment through sequence weighting, position-specific gap penalties and weight matrix choice. Nucleic Acids Research. 1994; 22:4673-4680

38. Parks DH, Rinke C, Chuvochina M, Chaumeil PA, Woodcroft BJ, Evans PN, et al. Recovery of nearly 8,000 metagenome-assembled genomes substantially expands the tree of life. Nat Microbiol. 2017;2:1533-1542

39. Price MN, Dehal PS, Arkin AP. FastTree 2 - approximately maximum-likelihood trees for large alignments. PLoS ONE. 2010;5:e9490.

40. Ludwig W, Strunk O, Westram R, Richter L, Meier H, Yadhukumar, et al. ARB: a software environment for sequence data. Nucleic Acids Res. 2004;32:1363-71.

41. Ashida H, Tamaki H, Fujimoto T, Yamamoto K, Kumagai H. Molecular cloning of cDNA encoding alpha$\mathrm{N}$-acetylgalactosaminidase from Acremonium sp. and its expression in yeast. Arch Biochem Biophys. 2000;384:305-10.

42. Balch WE, Fox GE, Magrum LJ, Woese CR, Wolfe RS. Methanogens: reevaluation of a unique biological group. Microbiol Rev. 1979;43:260-96.

43. Jarrell KF, Jones GM, Nair DB. Biosynthesis and role of $\mathrm{N}$-linked glycosylation in cell surface structures of archaea with a focus on flagella and $\mathrm{S}$ layers. Int $\mathrm{J}$ Microbiol. 2010;2010:20.

44. Magidovich H, Eichler J. Glycosyltransferases and oligosaccharyltransferases in Archaea: putative components of the $\mathrm{N}$ glycosylation pathway in the third domain of life. FEMS Microbiol Lett. 2009;300:122-30.

45. Konstantinidis KT, Ramette A, Tiedje JM. The bacterial species definition in the genomic era. Philos Trans R Soc B: Biol Sci. 2006;361:1929-40.

46. Kim M, Oh HS, Park SC, Chun J. Towards a taxonomic coherence between average nucleotide identity and 16S rRNA gene sequence similarity for species demarcation of prokaryotes. Int J Syst Evolut Microbiol. 2014;64:346-51.

47. Cayrou C, Henrissat B, Gouret P, Pontarotti P, Drancourt M. Peptidoglycan: a post-genomic analysis. BMC Microbiol. 2012;12:294.

48. Engelhardt Wv, Wolter S, Lawrenz H, Hemsley JA. Production of methalne in two non-ruminant herbivores. Comp Biochem Physiol Part A: Physiol. 1978;60:309-11.

49. Pinares-Patiño CS, Ebrahimi SH, McEwan JC, Dodds KG, Clark $\mathrm{H}$, Luo D. Is rumen retention time implicated in sheep differences in methane emission? Proceedings of the New Zealand Society of Animal Production. 2011;71:219-222

50. Moeller AH, Caro-Quintero A, Mjungu D, Georgiev AV, Lonsdorf EV, Muller MN, et al. Cospeciation of gut microbiota with hominids. Science. 2016;353:380. 